\title{
Reconsidering Primary HPV Testing in Cervical Cancer Screening
}

\author{
Carlo Antonio Liverani \\ Gynecologic Oncology Unit, Department of Mother and Infant Sciences, University of Milan, Milan 20122, Italy
}

Received: February 15, 2015 / Accepted: February 28, 2015 / Published: February 28, 2015.

\begin{abstract}
Inappropriate testing for HPV types on healthy subjects increases costs without benefit and potentially results in overtreatment. HPV testing also has a negative psychosocial impact on women, increasing anxiety, stress, and concerns on sexual relationships. Giving the fact that HPV testing has been shown to have similar sensitivity but more overdiagnosis than cytology, and also giving the fact that false negative results may be higher than previously suspected, primary screening with HPV tests in European countries should be reconsidered. Resources saved in molecular testing may well be addressed in implementing vaccination strategies which are still underused, and may possibly include males as well as women.
\end{abstract}

Key words: Human papillomavirus, cervical cancer, screening, HPV vaccine.

\section{Introduction}

In primary cervical cancer screening, many countries are being challenged to choose between cytology and HPV test (the latter to be performed alone or in combination with cytology, so called co-testing) [1]. But many health professionals lack sufficient knowledge about the epidemiology of HPV infection, its causal link with cervical cancer, and test management [2]. One of the consequences of this situation is the common request of useless and potentially harmful HPV tests, leading to a huge rise in health care costs and anxiety in the population. Inappropriate testing increases costs without benefit and potentially results in overtreatment of women [3, 4].

This problem has reached such proportions to induce the major American medical associations to state precise and shared guidelines regarding cervical cancer screening $[5,6]$, to address appropriate use of HPV testing. On the opposite side, the Japanese [7] and Canadian [8] guidelines do not recommend

Corresponding author: Carlo Antonio Liverani, M.D., research fields: HPV associated pathology, HPV vaccinations, colposcopy, gynecologic oncology, lower genital tract disesases. E-mail: dr.carlo.liverani@gmail.com. screening through testing for HPV.

In any case, the interpretation of a positive HPV result implies the knowledge of the natural history of HPV infection [9, 10]. An HPV positive, cytology negative woman, should repeat both tests after one year interval. Actually, daily clinical experience shows that too often patients tend to undergo immediate colposcopic examination. In this occasion, health care providers have the duty to counsel and inform patients on the frequent regressive nature of an HPV positive state, but at the same time they are telling a woman that:

- She is infected with an oncogenic virus;

- It is a "high risk" type;

- There are no visible lesions;

- There is no cure;

- It is sexually transmitted.

HPV testing can have important social and psychological effects on women and can potentially damage their wellbeing particularly when used outside an organized prevention program. The magnitude of these effects depends on the characteristics of the woman concerned, her prior knowledge of HPV, the communication content and skills of the health 
professional [11]. Negative psychosocial responses to HPV diagnosis, include: anxiety; fear; anger; shame or stigma; regret; overestimation of cancer risk; concerns about loss of reproductive functions; concerns about negative reactions from friends, family, or sexual partners; concerns about partner infidelity or hostility; changes in physical intimacy activities; sexual refusal; isolation; urgent need for treatment; depression [12, 13]. Factual uncertainties about HPV can not be resolved through the provision of more information [14]. HPV positivity may also pose a prolonged psychosocial burden on women even after having had the necessary follow-up for their cervical abnormalities [15].

This is particularly important when talking of prevention [16, 17]. Excessive preventive measures may lead to increased fear and perception of illness where none exists, and clinicians' frustration over a growing list of requirements that are impossible to accommodate. Clinicians will initiate multiple preventive, diagnostic, and therapeutic activities, each of which leads to interventions with well documented but poorly recognized ill effects. Eventually they tend to impute disease and risk factors to healthy individuals, and turn people into patients, without any evidence of benefit to them individually [18].

Studies on young women initiating first sexual activity suggest that nearly $30 \%$ of subjects test HPV DNA positive within 1 year of first intercourse with a first male sex partner, and by 3 years almost half test positive. The cumulative transition probability from an HPV DNA negative state to an HPV 16 DNA positive state is greater than $30 \%$ after 24 months of first sexual exposure [19]. With prolonged follow-up and frequent testing, HPV infections may be detected in nearly all adolescent women $(87 \%$ with a vaccine type). Infections with high risk types are characterized by longer duration, with periods of non-detections, suggesting that some infections may persist at low levels [20]. But the vast majority of HPV infections regresses within two years in more than $90 \%$ of cases; about $60 \%$ of women testing HPV DNA positive will develop specific serum antibodies against that particular HPV type, and if cellular samples are taken during the peak of productive viral infection, mild cytological abnormalities may be detected on cervical smears. It is known that treatment is not recommended for CIN 1 (cervical intraepithelial neoplasia grade 1), giving both the high rate of spontaneous regression as well as persistence after treatment [21]. However, even for low-grade abnormal cervical tests, women tend to prefer active management strategies; and it is very difficult to meet their expectations of optimal follow-up when HPV testing is used in primary screening [22].

Finally, HPV may remain in a latent state for years before causing disease [23], so that it is usually not possible to determine when and from whom initial infection was acquired. Most incident HPV infections are attributable to past, not current, sexual behavior, supporting a natural history model of viral latency and reactivation [24].

\section{Background Studies}

In 2009 Kitchener and coll. found that co-testing with HPV test and cytology was not able to detect a higher rate of CIN 2 or worse than liquid-based cytology alone (ARTISTIC trial) [25]. Moreover 3 out of 12 cervical cancers diagnosed in the first two rounds of the trial were preceded by negative baseline HPV testing (25\%). This false negative rate is similar to that observed by Cotton and coll. who showed that $22 \%$ of women who had CIN 2 or worse were HPV negative at baseline (TOMBOLA trial) [26]. It is difficult to accept these figures when one talks of extending rescreening intervals such as those proposed by HPV testing algorithms.

In 2013 Malila and coll. warned that the sensitivity of HPV testing is similar to that of cytology but causes more overdiagnosis [27]. In another study, Patanwala and coll. concluded that after two rounds of screening HPV testing based strategies were more 
sensitive than cytology for the detection of CIN 3 or worse only when referral to colposcopy followed a single positive HPV test (a strategy which is not recommended) [28]. In 2014 Elfström and coll. found that the cumulative incidence of CIN 2 or worse was the same for HPV screening and for cytology over long term follow-up, implying that the increased sensitivity of HPV screening for CIN 2 or worse reflects earlier detection rather than overdiagnosis [29]. Nevertheless early diagnosis may lead to overtreatments, especially because gynecologists do not always follow recommended guidelines.

There are many different HPV assays produced by different companies, but only few of them are validated. In one study evaluating the concordance among four of these validated tests, the disagreement was considerable. Among more than five thousand samples $33.2 \%$ tested positive on at least one of the four assays. Globally $41 \%$ of them tested positive on all four, but this figure decreased to only $29 \%$ in primary screening samples. In women with abnormal cytology the agreement was $68 \%$, thus implying that referral to colposcopy would depend on which of the four assays has been used in $32 \%$ of the cases. In HPV positive, cytology negative women, who represent the vast majority of HPV positive cases, the disagreement was even larger. These patients should be considered at higher risk, and a prolonged rescreening interval would not be advisable; but at the same time it is known that this risk is still relatively low, and a 6-12 months retesting would lead to repeated visits in a large group of women who might have tested negative had they been screened with another HPV assay [30].

\section{Cervical Cancer Screening in Europe}

The most relevant European study is a paper designed to evaluate the efficacy of HPV-based screening in four European randomised controlled trials, which has shown how the cumulative probability of developing cervical cancer was higher in women allocated to Pap smear in comparison with those allocated to HPV testing [1]. The study has been criticized, because none of the four trials showed a true reduction in cervical cancer incidence. Even after the data were pooled, the diagnostic rates were similar in the HPV arm and in the cytology arm during the first 2.5 years after enrolment. Thus the authors eliminated 52 out of 107 cases with the explanation that cancers diagnosed during the first 2.5 years represented mainly prevalent cases [31]. In the 55 remaining cases (almost half of the study population), 19 cancers were diagnosed in 419,000 women in the HPV arm, and 36 cancers in 358,656 women in the cytology arm, leading the authors to conclude that HPV-based screening is supposed to provide $60-70 \%$ greater protection against cervical cancers compared with cytology [1]. Furthermore, $40 \%$ of the total cases were represented by the Italian study, where all women were referred to colposcopy in case of a positive HPV result, with over double number of biopsies in the HPV arm compared to the cytology arm [31]. Eventually only 11 of 19 cervical cancers detected over 2.5 years after enrolment, were HPV-positive at baseline. Even among presumed "prevalent" cases, $16 \%$ were HPV-negative at baseline, similarly to what observed in our previous study, showing a $14 \%$ false negative rate in women treated for histologically confirmed high grade cervical lesions [32]. Again extending cervical rescreening should not be considered a safe approach. Nonetheless in the Netherlands, five-yearly cytological screening for women aged 30-60 years will be replaced by primary HPV screening in 2016 . Not only, but these newly developed guidelines involve an extension of the screening interval from 5 to 10 years for HPV-negative women aged 40 or 50 years [33].

\section{Cervical Cancer Screening in the USA}

The results of the first prospective U.S. screening study to evaluate the performance of HPV primary screening (ATHENA study) support the use of HPV 
primary screening [34, 35]. Most recently, "interim" guidelines addressing the use of HPV testing alone as a primary screening approach have been published [36]. But these first guidelines are based on insufficient data about the behaviour of this strategy in clinical practice and may not reveal clinically useful [37]. Until further data and algorithm development will be available, it seems premature to adopt HPV testing alone as a valid screening approach [38]. The Clinical Guidelines Synopsys recently published by Volerman and Cifu on JAMA summarizes the recommended strategies and screening intervals, while the statement on HPV DNA test utilization by Davey and coll. emphasizes all the situations in which testing for HPV is not recommended [39]. Fig. 1 shows the most cost-effective algorithm supposed to be used in the USA [5, 6, 39, 40]. Indeed this algorithm is somewhat intricate and difficult to remember, and thus not always followed in clinical practice. As a matter of fact, the most common repeat interval following a negative HPV result is usually 12 months, across all years studied [41].

\section{Cervical Cancer Screening in Canada, Japan, and Australia}

In Canada, Japan, and Australia, cervical cancer screening is programmed with cervical cytology alone.

According to the Canadian guidelines, recommendations do not address screening with HPV test because of insufficient data on mortality and incidence of invasive cancer: Cervical cytology every 3 years is recommended for women aged 25-69 years [8]. In Japan cervical cancer screening using either

\section{Cervical Cancer Screening in the USA}

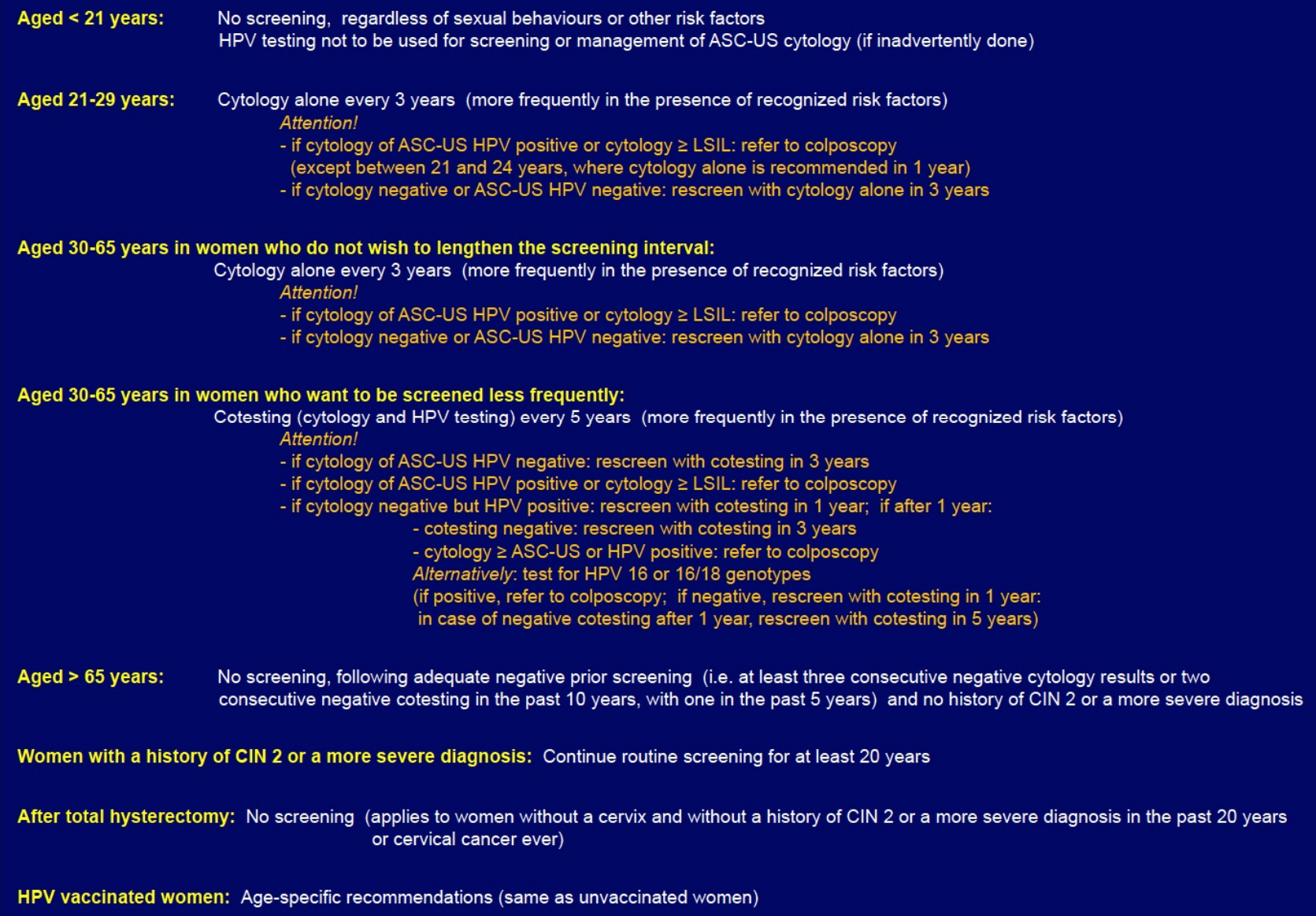

Fig. 12013 Cervical Cancer Screening Guidelines in the USA. 
HPV alone or co-testing is not recommended for population-based screening due to insufficient evidence: Cytology is recommended every 2 years starting at age 20 [7]. Similarly in Australia cytology is recommended every 2 years for women aged 20-69 years, and the National Cervical Screening Program will not be implemented prior to May 2017 [42, 43].

\section{Conclusion}

Giving the fact that HPV testing has been shown to have similar sensitivity but more overdiagnosis than the Pap test [25-28], and also giving the fact that false negative results may be higher than previously suspected $[25,26,32]$, primary screening with HPV testing in European countries as well as in the U.S. should be reconsidered. Resources saved in molecular testing may well be addressed in implementing vaccination strategies that are still underused and possibly must include males as well as women [44, 45].

In daily clinical practice, what many health professionals actually do is:

- Use non validated tests, as well as mRNA, FISH;

- Test women under 30 years (not recommended);

- Re-screen every 1-2 years (not recommended);

- Test for low risk types (not recommended);

- Use genotyping even when it's not indicated;

- Test anal, vulvar, penile, oral sites (not recommended);

- Test for low grade as well as for high grade lesions (which has no role in clinical management);

- Test male partner (not recommended);

- Test to screen for sexually transmitted infections (not recommended).

Eventually, the sensitivity of cytology in well established settings is over $80 \%[46,47]$. And so the main prerequisite to shift from cytology to HPV testing - that sensitivity of conventional Pap testing is only around $50 \%$ - simply is not true. But the worst consequences are that too many doctors start treating HPV infections detected with molecular tests with surgery, laser, cryotherapy, interferon, 5-florouracil, etc. Individual labelled as being at risk undergo a series of unnecessary diagnostic and surgical procedures [48].

Most recently, a paper designed to evaluate cervical cancer screening strategies over more than 250,000 samples from women aged 30 to 65 years who had a co-test and a cervical biopsy, found a rate of false negative results in $19 \%$ of women who had an HPV-only cervical screen and in $12 \%$ of those who had a Pap-only testing. Surprisingly, $26.6 \%$ of cervical adenocarcinomas were HPV negative, while 20.7\% were Pap test negative [49].

Eventually, it should not be forgotten that in the case of the ACS (American Cancer Society), ASCCP (American Society for Colposcopy and Cervical Pathology), ASCP (American Society for Clinical Pathology), approximately $25 \%$ of committee members reported financial associations with companies that make HPV tests, including the provision of research supplies and research funding, advisory and/or consulting roles, and speakers' honoraria [50]. Clinicians must be aware of the risk of being influenced by guidelines designed by commercially supported "consensus" groups that are reviewing the definition and management of diseases [51]. Broadening the boundaries of illness will lead to a waste of resources, raise in costs and anxiety, and the risk of under-recognition of true disease.

\section{Conflict of Interest Statement}

The author declares no conflict of interest.

\section{References}

[1] Ronco, G., Dillner, J., Elfström, K. M., Tunesi, S., Snijders, P. J., and Arbyn, M. et al. 2014. "Efficacy of HPV-Based Screening for Prevention of Invasive Cervical Cancer: Follow-up of Four European Randomised Controlled Trials." Lancet 383 (9916): 524-32.

[2] Mazzadi, A., Paolino, M., and Arrossi, S. 2012. "HPV Vaccine Acceptability and Knowledge among Gynecologists in Argentina." Salud Publica Mex 54 (5): 
515-22.

[3] Solomon, D., Papillo, J. L., and Davey, D. D. 2009. "Cytopathology Education and Technology Consortium Statement on HPV DNA Test Utilization." Am J Clin Pathol 131:768-9.

[4] Lee, J. W. Y., Berkowitz, Z., and Saraiya, M. 2011. "Low-Risk Human Papillomavirus Testing and Other Nonrecommended Human Papillomavirus Testing Practices among U.S. Health Care Providers." Obstet Gynecol 118 (1): 4-13.

[5] Moyer, V. A. 2012. "Screening for Cervical Cancer: U.S. Preventive Services Task Force Recommendation Statement." Ann Intern Med 156 (12): 880-91.

[6] Saslow, D., Solomon, D., Lawson, H. W., Killackey, M., Kulasingam, S. L., and Cain, J. et al. 2012. "American Cancer Society, American Society for Colposcopy and Cervical Pathology, and American Society for Clinical Pathology Screening Guidelines for the Prevention and Early Detection of Cervical Cancer." CA Cancer J. Clin. 62 (3): 147-72.

[7] Hamashima, C., Aoki, D., and Miyagi, E. 2010. "The Japanese Guideline for Cervical Cancer Screening." Jpn J. Clin. Oncol. 40 (6): 485-502.

[8] Canadian Task Force on Preventive Health Care, Dickinson, J., Tsakonas, E., Conner, G. S., Lewin, G., Shaw, E., and Singh, H. et al. 2013. "Recommendations on screening for Cervical Cancer." CMAJ 185 (1): 35-45.

[9] Gravitt, P. E. 2011. "The Known Unknowns of HPV Natural History.” J. Clin. Invest 121 (12): 4593-9.

[10] Liverani, C. A. 2013. "The Four Steps in the Prevention of Human Papillomavirus-Associated Neoplasia: Considerations for Preventive Measures, Screening, Disease Impact, and Potential Overtreatments in HPV-related Pathology." Arch Obstet Gynecol 288 (5): 979-88.

[11] Kahn, J. A., Slap, G. B., and Bernstein, D. I. 2005. "Psychological, Behavioral and Interpersonal Impact of Human Papillomavirus and Pap Test Results." J. Womens Health 14 (7): 650-9.

[12] Arrossi, S., and Wiesner, C. 2012. "The Psychological and Social Costs of HPV Testing in Screening Activities." HPV Today 27 (11): 4.

[13] McCaffery, K., Waller, J., and Forrest, S. 2004. "Testing Positive for Human Papillomavirus in Routine Cervical Screening: Examination of Psychosocial Impact.” BJOG 111 (12): 1437-43.

[14] Rosen, N. O., Knäuper, B., and Di, D. P. 2010. "The Impact of Intolerance of Uncertainty on Anxiety after Receiving an Informational Intervention about HPV: A Randomised Controlled Study." Psychol Health 25 (6): 651-68.

[15] Kwan, T. T., Cheung, A. N., and Lo, S. S. "Psychological
Burden of Testing Positive for High-Risk Human Papillomavirus on Women with Atypical Cervical Cytology: A Prospective Study." Acta Obstet Gynecol Scand 90 (5): 445-51.

[16] Moyer, V. A. 2012. "What We don't Know can Hurt Our Patients: Physician Innumeracy and Overuse of Screening Tests." Ann Intern Med 156: 392-3.

[17] Moynihan, R., Doust, J., and Henry, D. 2012. "Preventing Overdiagnosis: How to Stop Harming the Healthy." BMJ 344: e3502 doi: 10.1136.

[18] Gérvas, J., Starfield, B., and Heath, I. 2008. "Is Clinical Prevention Better than Cure?" Lancet 372: 1997-1999.

[19] Winer, R. L., Feng, Q., and Hughes, J. P. "Risk of Female Human Papillomavirus Acquisition Associated with First Male Sex Partner." J Infect Dis 197 (2): 279-82.

[20] Shew, M., Ermel, A., and Weaver, B. 2011. "HPV Detection from Adolescent Women with Prolonged Follow-up." In Proceedings of the $27^{\text {th }}$ International Papillomavirus Conference, 160.

[21] Centers for Disease Control and Prevention. 2010. "Sexually Transmitted Diseases Treatment Guidelines." Recommendations and Reports December 17, MMWR 59 (No. RR-12): 69-78.

[22] Frederiksen, M. E., Lynge, E., and Rebolj, M. 2012. "What Women Want Women's Preferences for the Management of Low-Grade Abnormal Cervical Screening Tests: A Systematic Review." BJOG 119 (1): 17-9.

[23] Maglennon, G. A., McIntosh, P., and Doorbar, J. 2011. "Persistence of Viral DNA in the Epithelial Basal Layer Suggests a Model for Papillomavirus Latency Following Immune Regression.” Virology 414 (2): 153-63.

[24] Rositch, A. F., Burke, A. E., and Viscidi, R. P. 2012. "Contributions of Recent and Past Sexual Partnerships on Incident Human Papillomavirus Detection: Acquisition and Reactivation in Older Women." Cancer Res 72 (23): 6183-90.

[25] Kitchener, H. C. 2009. "HPV Testing in Combination with Liquid-Based Cytology in Primary Cervical Screening (ARTISTIC): A Randomised Controlled Trial." Lancet Oncol 10 (7): 672-82.

[26] Cotton, S., Sharp, L., and Little, J. 2010. "Trial Of Management of Borderline and Other Low-grade Abnormal Smears Group. The Role of Human Papillomavirus Testing in the Management of Women with Low-grade Abnormalities: Multicentre Randomised Controlled Trial." BJOG 117 (6): 645-59.

[27] Malila, N., Leinonen, M., and Kotaniemi-Talonen, L. 2013. "The HPV Test Has Similar Sensitivity but More Overdiagnosis than the Pap Test: A Randomised Health Services Study on Cervical Cancer Screening in Finland." Int. J. Cancer 132 (9): 2141-7. 
[28] Patanwala, I. Y. 2013. "A Systematic Review of Randomized Trials Assessing Human Papillomavirus Testing in Cervical Cancer Screening." Am J Obstet Gynecol 208 (5): 343-53.

[29] Elfström, K. M., Smelov, V., Johansson, A. L., Eklund, C., Nauclér, P., and Arnheim-Dahlström, L. et al. 2014. "Long Term Duration of Protective Effect for HPV Negative Women: Follow-Up of Primary HPV Screening Randomised Controlled Trial." BMJ 348: 130.

[30] Rebolj, M., Preisler, S., and Ejegod, D. M. 2014. "Disagreement between Human Papillomavirus Assays: An Unexpected Challenge for the Choice of an Assay in Primary Cervical Screening." PLoS One 9 (1): e86835. doi: 10.1371 .

[31] Austin, R. M. 2014. "Can HPV Primary Screening Reduce Cervical Cancer Incidence and Mortality?" SCAN 25 (1): 7-8.

[32] Liverani, C. A., Ciavattini, A., and Monti, E. 2012. "High Risk HPV DNA Subtypes and E6/E7 mRNA Expression in a Cohort of Colposcopy Patients from Northern Italy with High-grade Histologically Verified Cervical Lesions." Am J Transl Res 4 (4): 452-7.

[33] Vink, M. A., Bogaards, J. A., Meijer, C. J., and Berkhof, J. 2014. "Primary Human Papillomavirus DNA Screening for Cervical Cancer Prevention: Can the Screening Interval Be Safely Extended?" Int J Cancer 12 (6). doi: 10.1002/ijc.29381.

[34] Cox, J. T., Castle, P. E., and Behrens, C. M. 2013. "Comparison of Cervical Cancer Screening Strategies Incorporating Different Combinations of Cytology, HPV Testing, and Genotyping for HPV 16/18: Results from the ATHENA HPV Study.” Am J Obstet Gynecol 208 (184): e1-11.

[35] Wright, T. C., Stoler, M. H., Behrens, C. M., Sharma, A., Zhang, G. L., and Wright, T. L. W. 2015. "Primary Cervical Cancer Screening with Human Papillomavirus: End of Study Results from the ATHENA Study Using HPV as the First-Line Screening Test." Gynecol Oncol 136 (2): 189-97.

[36] Huh, W. K., Ault, K. A., and Chelmow, D. "Use of Primary High-Risk Human Papillomavirus Testing for Cervical Cancer Screening: Interim Clinical Guidance." Obstet Gynecol 125 (2): 330-7.

[37] Feldman, S. 2014. "Human Papillomavirus Testing for Primary Cervical Cancer Screening. Is It Time to Abandon Papanicolaou Testing?" JAMA Intern Med 174 (10): 1539-40.

[38] Volerman, A., and Cifu, A. S. 2014. "JAMA Clinical Guidelines Synopsis: Cervical Cancer Screening." JAMA
312 (21): 2279-80

[39] Davey, D. D., Goulart, R., and Nayar, R. 2014. "Cytopathology Education and Technology Consortium (CETC). 2013 Statement on Human Papillomavirus DNA Test Utilization." Diagn Cytopathol 42 (5): 449-52.

[40] Massad, L. S., Einstein, M. H., and Huh, W. K. 2013. "2012 Updated Consensus Guidelines for the Management of Abnormal Cervical Cancer Screening Tests and Cancer Precursors." Obstet Gynecol 121 (4): 829-46.

[41] Jackson, B. 2014. “Overtesting for Cervical Cancer: Patterns and Trends from a National Reference Laboratory in the United States." Presented at the 2014 Preventing Overdiagnosis Conference, Oxford, UK.

[42] Australian Institute of Health and Welfare 2015. Cervical screening in Australia 2012-2013. Cancer series no. 93. Cat. no. CAN 91. Canberra: AIHW.

[43] Renewal of the National Cervical Screening Program. Accessed May 27, 2015. http://www.cancerscreening.gov.au/internet/screening/pu blishing.nsf/Content/cervical-screening-1.

[44] Oscarsson, M. G., Hannerfors, A. K., and Tydén, T. 2012. "Young Women's Decision-making Process for HPV Vaccination." Sex Reprod Healthc 3 (4): 141-6.

[45] Jin, X. W., Lipold, L., Sikon, A., and Rome, E. 2013. "Human Papillomavirus Vaccine: SAFE, Effective, Underused." Cleve Clin J. Med 80 (1): 49-60.

[46] Nanda, K., McCrory, D. C., and Myers, E. R. 2000. "Accuracy of the Papanicolaou Test in Screening for and Follow-up of Cervical Cytologic Abnormalities: A Systematic Review." Ann Intern Med 132: 810-9.

[47] Hegde, D., Shetty, H., Shetty, P. K., and Rai, S. 2011. "Diagnostic Value of Acetic Acid Comparing with Conventional Pap Smear in the Detection of Colposcopic Biopsy-proved CIN." J. Cancer Res Ther 7: 454-58.

[48] Heath, I. 2013. "Overdiagnosis: When Good Intentions Meet Vested Interests.” BMJ 347: f6361.

[49] Blatt, A. J., Kennedy, R., Luff, R. D., Austin, R. M., and Rabin, D. S. 2015. "Comparison of Cervical Cancer Screening Results among 256, 648 Women in Multiple Clinical Practices." Cancer Cytopathol 123 (5): 282-8.

[50] Smith-McCune, K. 2014. "Choosing a Screening Method for Cervical Cancer Papanicolaou Testing alone or with Human Papillomavirus Testing." JAMA Published Online May 5, 2014. doi: 10.1001/jamainternmed.2014.1368.

[51] Doran, E., and Henry, D. 2008. "Disease Mongering: Expanding the Boundaries of Treatable Disease." Intern Med J. 38 (11): 858-61. 